УДК 502:504.54:911.2:911.52:519.876

ВЛИЯНИЕ 40 ФАКТОРОВ ЖИЗНИ НА ДОЛЮ НАСЕЛЕНИЯ НИЖЕ ПРОЖИТОЧНОГО МИНИМУМА В СУБЪЕКТАХ УРАЛА И СИБИРИ

Мазуркин П.М.

ФГБОУ ВО «Поволжский государственный технологический университет», Йошкар-Ола, е-таil: kaf_po@mail.ru

\begin{abstract}
Для 14 субъектов федерации Урала и Сибири была составлена иерархия из 40 влияющих переменных на показатель доли населения ниже прожиточного минимума. Этот показатель в факторном анализе получил первое место. В иерархии переменных первое место занимает фактор использования воды, второе - средние душевые доходы населения. На основе анализа кривых Лоренца и Кузнеца, а также коэффициента Джини по взаимному влиянию доходов населения Х37 на долю ниже прожиточного минимума Х33, показана возможность оценки критерия неравномерности распределения значений влияющих переменных по статистическим показателям стандартного отклонения фактических значений показателя от расчетной по двухкомпонентным трендам и асимметричным вейвлетам, а также по коэффициентам корреляции. Оказалось, что кривая Лоренца является в общем случае многокомпонентным уравнением, содержащим, кроме тренда, дополнительные колебания. Для пары Х37-Х33 получаем среднее арифметическое 17,1\%, при этом стандартное отклонение $2,400 \%$. Для среднеарифметического значения стандартное отклонение равно 6,165\%. Тогда коэффициент неравномерности для показателя Х33 будет равен 0,3893. Для пары Х33-Х37 будет среднеарифметическое 27535,1 руб/мес., а стандартное отклонение формулы 6284,3 руб/мес., причем стандартное отклонение среднеарифметического равно 8160,5 руб/мес. Тогда коэффициент неравномерности будет равен $6284,3 / 8160,5=0,7701$. В итоге Х37-Х33 в сравнении с Х33-Х37 в два раза предпочтительнее по коэффициенту неравномерного распределения. В статье доказана квантовая определенность параметров социумов в виде населения субъектов федерации. В дальнейшем возможна идентификация вейвлетов для всех субъектов федерации России.
\end{abstract}

Ключевые слова: 40 параметров, прожиточный минимум, парные отношения, тренды, рейтинг влияния

\title{
INFLUENCE OF 40 LIFE FACTORS ON POPULATION SHARE BELOW THE LIVING MINIMUM IN THE SUBJECTS OF THE URALS AND SIBERIA
}

\section{Mazurkin P.M.}

\author{
Volga State University of Technology, Yoshkar-Ola, e-mail:kaf po@mail.ru
}

\begin{abstract}
For 14 subjects of the federation of the Urals and Siberia, a hierarchy of 40 influencing variables was compiled for the indicator of the share of the population below the subsistence level. This indicator was ranked first in factor analysis. In the hierarchy of variables, the first place is taken by the factor of water use, the second is the average per capita income of the population. Based on the analysis of the Lorenz and Kuznets curves, as well as the Gini coefficient for the mutual influence of the incomes of the population X37 on the share below the subsistence minimum X33, the possibility of assessing the criterion of the uneven distribution of the values of influencing variables according to the statistical indicators of the standard deviation of the actual values of the indicator from the calculated one by two-component trends and asymmetric wavelets, as well as correlation coefficients. It turned out that the Lorentz curve is, in the general case, a multicomponent equation containing, in addition to the trend, additional fluctuations. For the X37-X33 pair, we get the arithmetic mean of $17.1 \%$, while the standard deviation is $2.400 \%$. For the arithmetic mean, the standard deviation is $6.165 \%$. Then the coefficient of unevenness for the X33 index will be equal to 0.3893 . For the pair X33-X37, the arithmetic mean is 27535.1 rubles / month, and the standard deviation of the formula is 6284.3 rubles / month, and the standard deviation of the arithmetic mean is 8160.5 rubles / month. Then the coefficient of unevenness will be equal to $6284.3 / 8160.5=0.7701$. As a result, $\mathrm{X} 37-\mathrm{X} 33$ in comparison with X33-X37 is two times more preferable in terms of uneven distribution coefficient. The article proves the quantum definiteness of the parameters of societies in the form of the population of the subjects of the federation. In the future, it is possible to identify wavelets for all subjects of the Russian Federation.
\end{abstract}

Keywords: 40 parameters, living wage of the population, pair relations, trends, influence rating

Колебательная адаптация существует в природе, включая живое и косное вещества по В.И. Вернадскому. Этот принцип адаптации действует и на социумы, например в виде субъектов федерации. Тогда получается, что закон Коммонера «всё связано со всем» исходит из взаимных колебаний процессов адаптации, которые проявляются в виде суммы асимметричных вейвлетов [1]. Коллективные действия необходимы, чтобы отвести земную систему от порога глобального экологического кризиса. Такие действия включают управление системой Земли - биосферой, климатом и обществами - на уровнях изменений поведения [2].

Доход населения также является важным фактором. Неравномерное распределение доходов оказывает давление на окружающую среду как со стороны самого низкого, так и самого высокого уровня доходов. Чтобы просто выжить, мно- 
гие из беднейших людей мира участвуют в нерациональном использовании ресурсов. Они также вынуждены истощать скудные природные ресурсы, такие как леса или популяции животных, чтобы прокормить свои семьи [3].

Экологические инновации в «зеленых» технологиях становятся основным фактором экономического роста, при этом происходит неравномерное развитие этих инноваций и технологий в разных странах мира [4]. В статье рассмотрены стратегические риски отставания России от ведущих в экономике стран.

Цель исследования - иерархия по коэффициенту корреляции трендов [1] влияния 40 параметров у 14 субъектов Урала и Сибири на долю населения ниже прожиточного минимума, методика коэффициента неравномерности Джини по кривой Лоренца, принятой в виде тренда.

\section{Материалы и методы исследования}

По исследованиям [5] за 2014-2019 гг., на протяжении четырех лет начиная с 2014 г., в России наблюдалось снижение реальных располагаемых денежных доходов населения. Коэффициент Джини уменьшился с 0,419 до 0,412, что свидетельствует о незначительном снижении неравенства доходов населения.

Иерархия влияюших переменных была составлена по результатам факторного анализа 40 параметров у 14 субъектов Урала и Сибири. Всего было получено 1600 уравнений в виде закономерности (1), из которых 1360 относятся к парным отношениям. Как зависимый показатель на первом месте оказался фактор X33 - доля населения ниже прожиточного минимума, \% (табл. 1). По иерархии уменьшения коэффициента корреляции как влияющая переменная на первом месте оказался фактор - использование воды, м $^{3} /$ чел. После воздуха потребление воды для человека является второй первичной потребностью.

Доля растительного покрова от суши почти не влияет на долю населения ниже прожиточного минимума. Однако [3] бедное население влияет на снижение растительности.

Таблица 1

Влияющие факторы на показатель Х33 - доля населения ниже прожиточного минимума, \%

\begin{tabular}{|c|c|c|c|}
\hline $\begin{array}{c}\text { Ранг } \\
R\end{array}$ & $\begin{array}{c}\text { Код } \\
x\end{array}$ & $\begin{array}{l}\text { Коэф. } \\
\text { кор. } r\end{array}$ & Наименование влияющего параметра \\
\hline 0 & $\mathrm{X} 33$ & 0,9940 & доля населения ниже прожиточного минимума (\%) по рангам \\
\hline 1 & $\mathrm{X} 19$ & 0,9527 & 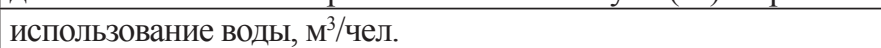 \\
\hline 2 & $\mathrm{X} 37$ & 0,9461 & средние душевые доходы населения, руб/мес. \\
\hline 3 & $\mathrm{X} 01$ & 0,9356 & приведенная северная широта центра столицы, $\alpha:=\alpha-50$ \\
\hline 4 & $\mathrm{X} 32$ & 0,9263 & уровень безработицы, \% \\
\hline 5 & $\mathrm{X} 28$ & 0,9141 & младенческая смертность на 1000 родившихся живыми \\
\hline 6 & $\mathrm{X} 27$ & 0,8944 & суммарный коэффициент рождаемости \\
\hline 7 & $\mathrm{X} 11$ & 0,8777 & сумма осадков за июль 2018 г., мм \\
\hline 8 & $\mathrm{X} 08$ & 0,8607 & средняя месячная температура в январе 2018 г., ${ }^{\circ} \mathrm{C}$ \\
\hline 9 & $\mathrm{X} 21$ & 0,8598 & выбросы в атмосферу, кг/чел. \\
\hline 10 & $\mathrm{X} 17$ & 0,8316 & общий экологический коэффициент \\
\hline 11 & $\mathrm{X} 36$ & 0,8310 & ВРП на душу населения за 2018 г., тыс. руб/чел. \\
\hline 12 & $\mathrm{X} 03$ & 0,8244 & высота центра столицы над уровнем Балтийского моря, м \\
\hline 13 & $\mathrm{X} 26$ & 0,8165 & ожидаемая продолжительность жизни женщин, лет \\
\hline 14 & $\mathrm{X} 30$ & 0,8104 & естественный прирост на 1000 населения \\
\hline 15 & $\mathrm{X} 14$ & 0,8100 & доля пашни к площади суши, \% \\
\hline 16 & $\mathrm{X} 40$ & 0,8086 & число умерших женщин в возрасте $16-54$ лет на $10^{5}$ населения \\
\hline 17 & $\mathrm{X} 24$ & 0,7999 & ожидаемая продолжительность жизни всех, лет \\
\hline 18 & $\mathrm{X} 07$ & 0,7996 & среднее число в месяц дней с осадками > 0,1 мм (1961-1990) \\
\hline 19 & $\mathrm{X} 31$ & 0,7800 & уровень занятости, \% \\
\hline 20 & X39 & 0,7717 & число умерших мужчин в возрасте 16-59 лет на $10^{5}$ населения \\
\hline 21 & $\mathrm{X} 35$ & 0,7528 & доля сельского населения, \% \\
\hline 22 & $\mathrm{X} 04$ & 0,7527 & средняя месячная ночная температура (1961-1990), ${ }^{\circ} \mathrm{C}$ \\
\hline 23 & $\mathrm{X} 16$ & 0,7432 & доля измененных человеком угодий к площади суши, \% \\
\hline 24 & X34 & 0,7388 & доля городского населения, \% \\
\hline
\end{tabular}




\begin{tabular}{|c|c|c|c|}
\hline & & & Окончание табл. 1 \\
\hline $\begin{array}{c}\text { Ранг } \\
R\end{array}$ & $\begin{array}{c}\text { Код } \\
x\end{array}$ & $\begin{array}{l}\text { Коэф. } \\
\text { кор. } r\end{array}$ & Наименование влияющего параметра \\
\hline 25 & $\mathrm{X} 25$ & 0,7142 & ожидаемая продолжительность жизни мужчин, лет \\
\hline 26 & $\mathrm{X} 22$ & 0,5907 & улавливание выбросов в атмосферу, кг/чел. \\
\hline 27 & $\mathrm{X} 10$ & 0,5677 & сумма осадков за январь 2018 г., мм \\
\hline 28 & $\mathrm{X} 20$ & 0,5647 & сброс загрязнений в воду, м³/чел. \\
\hline 29 & $\mathrm{X} 23$ & 0,5331 & плотность населения, чел/км² \\
\hline 30 & $\mathrm{X} 02$ & 0,5284 & приведенная восточная долгота, причем $\beta:=\beta-60,{ }^{\circ}$ \\
\hline 31 & X09 & 0,5241 & средняя температура в июле 2018 г., ${ }^{\circ} \mathrm{C}$ \\
\hline 32 & $\mathrm{X} 06$ & 0,5015 & средняя сумма осадков (1961-1990), мм \\
\hline 33 & $\mathrm{X} 05$ & 0,4922 & средняя дневная температура (1961-1990), ${ }^{\circ} \mathrm{C}$ \\
\hline 34 & $\mathrm{X} 38$ & 0,4697 & умершие оба пола в трудоспособном возрасте на $10^{5}$ населения \\
\hline 35 & $\mathrm{X} 18$ & 0,3959 & забор воды, м³/чел. \\
\hline 36 & $\mathrm{X} 29$ & 0,3931 & коэффициент миграционного прироста на $10^{4}$ населения \\
\hline 37 & $\mathrm{X} 12$ & 0,3681 & доля сельхозугодий к площади суши,\% \\
\hline 38 & $\mathrm{X} 13$ & 0,3059 & доля лесов к площади суши,\% \\
\hline 39 & $\mathrm{X} 15$ & 0,2479 & доля растительности «трава + кусты + деревья» к суше, \% \\
\hline
\end{tabular}

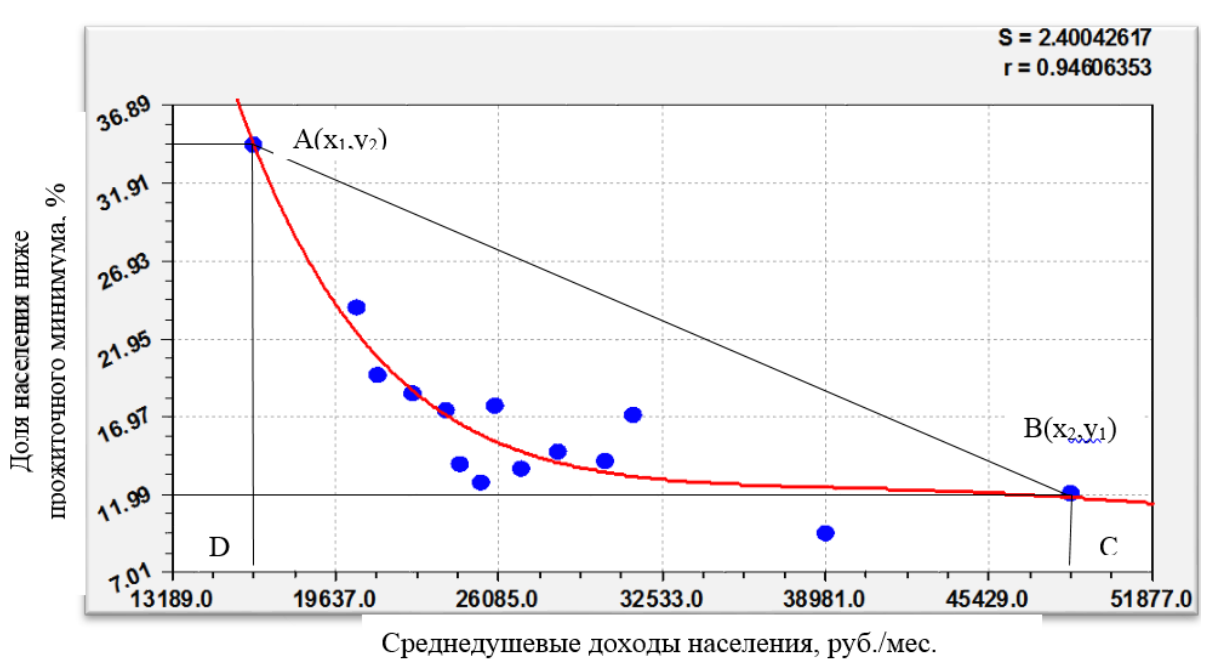

Рис. 1. Схема к расчету коэффиичента Джини неравномерного влияния среднедушевых доходов на долю населения ниже прожиточного минимума: $S$ - стандартное отклонение; $r$ - коэффициент корреляциии

Кривая Лоренца является методом графического изображения уровня концентрации явления. Математически эта кривая описывается трендом.

Все модели бинарных отношений были выявлены по формуле

$$
y=a \exp \left(-b x^{c}\right)+d x^{e} \exp \left(-f x^{g}\right),
$$

где $y$ - показатель, $x$ - влияющая переменная, $a-g-$ параметры (1) двухчленного тренда, идентифицируемые в среде CurveExpert-1.40. Второй член соответствует кривой Кузнеца.

Коэффициент Джини вычисляется относительно кривой Лоренца. При этом, если кривая Лоренца характеризуется общим уравнением двухсоставного тренда (1), то появляется возможность разработки методики расчета коэффициента Джини.

На рис. 1 приведен график уравнения (1) для влияния среднедушевых доходов населения на долю населения ниже прожиточного минимума для 14 субъектов федерации.

Кривой Лоренца становится график уравнения (1). Но, в отличие от общепринятой методики расчета неравномерности распределения мы примем не диагональ от 0 до 1 , а конкретные граничные значения (минимумы и максимумы) влияющей переменой $x$ на изменение зависимого показателя $y$. 
Тогда линия АВ будет линией равномерного распределения. Трапеция $\mathrm{ABCD}$ покажет теоретический объем равномерного распределения. В этом случае площадь под кривой Лоренца в интервале от $x_{1}$ до $x_{2}$ будет характеризовать фактический объем неравномерного распределения. Тогда отношение площади фигуры кривой Лоренца до прямой линии $\mathrm{AB}$ (числитель) к площади трапеции ABCD (знаменатель) станет коэффициентом Джини.

Рассмотрим обратное парное отношение (рис. 2) между этими же параметрами. Здесь картина усложнилась: второй слева субъект получил больший среднедушевой доход.

Опыт моделирования показал, что переход от точки $\mathrm{A}_{1}$ к более высокой по оси ор- динат точке А показывает волновой подъем значения показателя по асимметричному вейвлету.

\section{Результаты исследования и их обсуждение}

Для одного показателя и множества переменных в табл. 1 приведены 39 влияющих параметров на долю населения ниже прожиточного минимума. Из математической статистики известно, что в таком случае мерой неравномерности распределения становится стандартное отклонение $S$, которое автоматически выводится в программной среде CurveExpert-1.40.

В табл. 2 приведены параметры модели (1) для всех влияющих переменных.

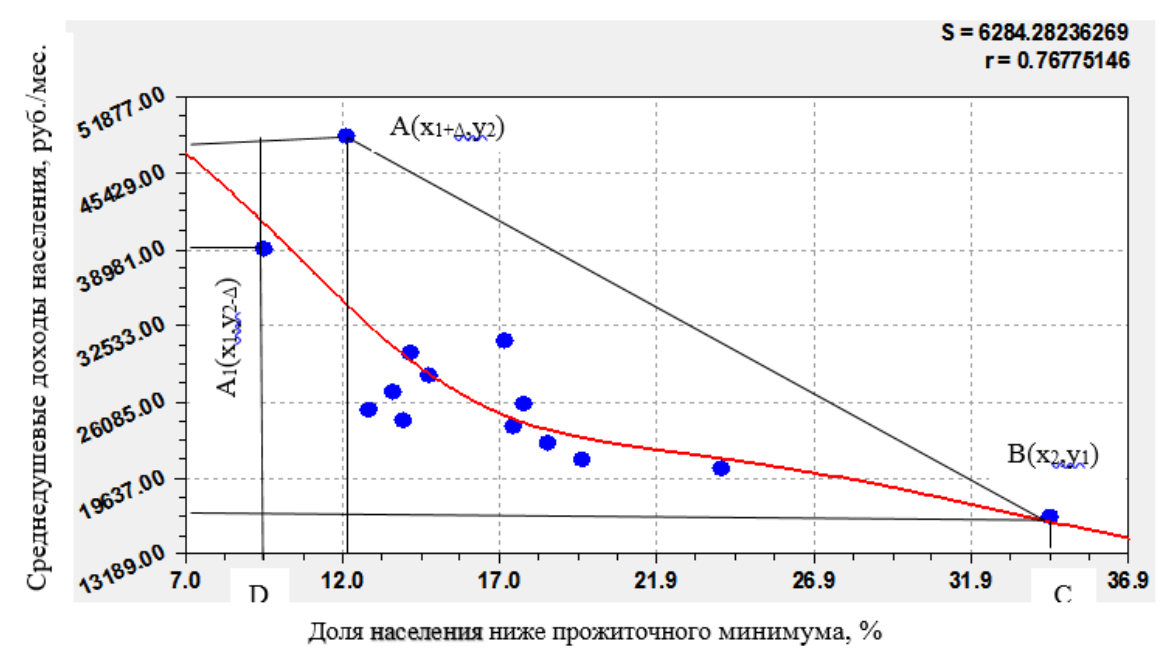

Рис. 2. Схема к расчету коэффиџиента Джини обратного влияния доли населения ниже прожиточного минимума на среднедушевые доходы

Таблица 2

Параметры тренда (1) доли населения ниже прожиточного минимума и стандартное отклонение от влияния разных переменных

\begin{tabular}{|c|c|c|c|c|c|c|c|c|c|c|}
\hline Ранг & Код & Коэф. & \multicolumn{2}{|c|}{ Экспоненциальный закон } & \multicolumn{3}{|c|}{ Биотехнический закон } & Станд. \\
\cline { 5 - 12 } & $x$ & кор. $r$ & $a$ & $b$ & $c$ & $d$ & $e$ & $f$ & $g$ & откл. $S$ \\
\hline 1 & $\mathrm{X} 19$ & 0,9527 & 247,1966 & 0,00084935 & 1 & $-173,4034$ & 0,057818 & 0,0010665 & 1 & 2,251 \\
\hline 2 & $\mathrm{X} 37$ & 0,9461 & 516,1222 & $4,54980 \mathrm{e}-5$ & 1 & $-3,31655$ & 0,53959 & $6,62654 \mathrm{e}-5$ & 1 & 2,400 \\
\hline 3 & $\mathrm{X} 01$ & 0,9356 & $1,96104 \mathrm{e} 6$ & 4,46699 & 0,30697 & $-4,04202 \mathrm{e} 6$ & 0,16996 & 5,20013 & 0,29277 & 2,966 \\
\hline 4 & $\mathrm{X} 32$ & 0,9263 & 11,06136 & $-0,021548$ & 1 & 0,039664 & 2,29383 & 0 & 0 & 2,649 \\
\hline 5 & $\mathrm{X} 28$ & 0,9141 & 41,09944 & 0,30629 & 1 & 0,037217 & 2,99788 & 0 & 0 & 2,850 \\
\hline 6 & $\mathrm{X} 27$ & 0,8944 & 3,88416 & $-0,834432$ & 0,88638 & 32,52691 & 9,90786 & 2,51978 & 2,50719 & 3,757 \\
\hline 7 & $\mathrm{X} 11$ & 0,8777 & 16,44231 & $2,74932 \mathrm{e}-5$ & 2,06631 & 0,064596 & 11,53535 & 0,017520 & 1,06918 & 4,026 \\
\hline 8 & $\mathrm{X} 08^{1}$ & 0,8607 & $9,99330 \mathrm{e} 5$ & 1,16173 & 0,58811 & $-1,03564 \mathrm{e} 6$ & 0,25614 & 1,40071 & 5,63718 & 4,277 \\
\hline 9 & $\mathrm{X} 21$ & 0,8598 & 21968,99 & 0,0022284 & 1 & $-21910,02$ & 0,0005339 & 0,0022341 & 1 & 3,784 \\
\hline 10 & $\mathrm{X} 17$ & 0,8316 & 16,49388 & 0,032043 & 1 & 0,0097469 & 2,00118 & 0 & 0 & 3,904 \\
\hline 11 & $\mathrm{X} 36$ & 0,8310 & 28707,78 & 0,0014637 & 1 & $-22188,86$ & 0,0044737 & 0,0014756 & 1 & 4,122 \\
\hline 12 & $\mathrm{X} 03$ & 0,8244 & 13,99281 & $-0,0006371$ & 1 & $8,6131 \mathrm{e}-38$ & 13,66546 & 0 & 0 & 3,978 \\
\hline 13 & $\mathrm{X} 26$ & 0,8165 & 705,53701 & 0,00060270 & 1 & $-8,04954$ & 1,23451 & 0,012435 & 1 & 4,278 \\
\hline
\end{tabular}




\begin{tabular}{|c|c|c|c|c|c|c|c|c|c|c|}
\hline \multirow{3}{*}{$\begin{array}{c}\text { Ранг } \\
R \\
\end{array}$} & \multirow{3}{*}{$\begin{array}{c}\text { Код } \\
x \\
\end{array}$} & \multirow{3}{*}{$\begin{array}{l}\text { Коэф. } \\
\text { кор. } r\end{array}$} & \multicolumn{8}{|c|}{ Окончание табл. 2} \\
\hline & & & \multicolumn{3}{|c|}{ Экспоненциальный закон } & \multicolumn{4}{|c|}{ Биотехнический закон } & \multirow{2}{*}{$\begin{array}{l}\text { Станд. } \\
\text { откл. } S\end{array}$} \\
\hline & & & $a$ & $b$ & $c$ & $d$ & $e$ & S & $g$ & \\
\hline 14 & $\mathrm{X} 30^{2}$ & 0,8104 & 14,65075 & $-0,0090517$ & 1 & $8,2048 \mathrm{e}-28$ & 21,65281 & 0 & 0 & 4,118 \\
\hline 15 & $\mathrm{X} 14$ & 0,8100 & 436,9968 & 0,20190 & 0,56916 & $-566,8485$ & 0,21060 & 0,55589 & 0,44460 & 4,927 \\
\hline 16 & $\mathrm{X} 40$ & 0,8086 & 13,89734 & $-0,00050076$ & 1 & $7,703 \mathrm{e}-115$ & 44,75396 & 0 & 0 & 4,135 \\
\hline 17 & $\mathrm{X} 24$ & 0,7999 & 1034,8541 & $-0,0055406$ & 1 & $-10,76146$ & 1,36119 & 0,012008 & 1 & 4,447 \\
\hline 18 & $\mathrm{X} 07$ & 0,7996 & 77,87132 & $-0,00026476$ & 1,57491 & $-5,33149$ & 0,63472 & 0 & 0 & 4,449 \\
\hline 19 & $\mathrm{X} 31$ & 0,7800 & 495,48864 & 0,0097055 & 1 & $-0,52297$ & 2,16570 & 0,044095 & & 4,636 \\
\hline 20 & $\mathrm{X} 39$ & 0,7717 & 91,76271 & 0,00057999 & 1 & $75,81 \mathrm{e}-136$ & 45,34476 & 0 & 0 & 2,939 \\
\hline 21 & $\mathrm{X} 35$ & 0,7528 & $7,81816 \mathrm{e} 10$ & 1,69061 & 1 & 0,35638 & 1,30429 & 0,022285 & 1 & 4,877 \\
\hline 22 & $\mathrm{X} 04^{3}$ & 0,7527 & $8,77390 \mathrm{e}-8$ & $-17,06038$ & 0,044176 & $-4,7959 \mathrm{e}-5$ & 7,52045 & 4,38224 & 0,33150 & 5,531 \\
\hline 23 & $\mathrm{X} 16$ & 0,7432 & 32655,89 & 1,48449 & 0,17180 & \begin{tabular}{|l|}
$-4,28887,4$ \\
\end{tabular} & 0,034072 & 1,76232 & 0,16206 & 5,621 \\
\hline 24 & $\mathrm{X} 34$ & 0,7388 & 13,63130 & $-0,026993$ & 1 & $-0,0005945$ & 2,75619 & 0 & 0 & 4,736 \\
\hline 25 & $\mathrm{X} 25$ & 0,7142 & 584,41677 & $-0,0012761$ & 1 & $-7,25376$ & 1,29477 & 0,019788 & & 5,186 \\
\hline 26 & $\mathrm{X} 22$ & 0,5907 & 26,75275 & $-0,00023247$ & 1 & $-1,13134$ & 0,41979 & 0 & 0 & 5,672 \\
\hline 27 & $\mathrm{X} 10$ & 0,5677 & 23,19547 & 0,011778 & 1 & $-5,749 \mathrm{e}-15$ & 0,23589 & 1,96318 & 1 & 6,099 \\
\hline 28 & $\mathrm{X} 20$ & 0,5647 & 23,22734 & $-0,0029644$ & 1 & $\begin{array}{l}-0,29644 \\
\end{array}$ & 0,96725 & 0 & 0 & 5,801 \\
\hline 29 & $\mathrm{X} 23$ & 0,5331 & 21,55956 & $-0,024 \varepsilon$ & 1 & $-0,99309$ & 1,03409 & 0 & 0 & 5,947 \\
\hline 30 & $\mathrm{X} 02$ & 0,5284 & 5,14063 & $-0,016437$ & 1 & 6,39911 & 0,15393 & 0 & ( & 5,968 \\
\hline 31 & $\mathrm{X} 09$ & 0,5241 & 0,69175 & $-0,32229$ & 1 & $-1,6362 \mathrm{e}-6$ & 6,46521 & 0 & 0 & 5,986 \\
\hline 32 & $\mathrm{X} 06$ & 0,5015 & 33,05282 & 0 & 0 & $\begin{array}{l}-0,078449 \\
\end{array}$ & 0,87912 & 0 & 0 & 5,798 \\
\hline 33 & $\mathrm{X} 05$ & 0,4922 & 1,45113 & 0,063030 & 1 & $-3,6539 \mathrm{e}-9$ & 5,65311 & 0 & 0 & 6,118 \\
\hline 34 & $\mathrm{X} 38$ & 0,4697 & $-0,0040772$ & 0,00028413 & 1 & 0,0086862 & 1,20059 & 0 & 0 & 6,205 \\
\hline 35 & $\mathrm{X} 18$ & 0,3959 & 6723,2324 & 0,0015142 & 1 & $-6675,1053$ & 0,0010532 & 0,0015231 & 1 & 6,804 \\
\hline 36 & $\mathrm{X} 29^{4}$ & 0,3931 & 11,81876 & $-0,027780$ & 1 & $-0,037033$ & \begin{tabular}{|l|}
1,48044 \\
\end{tabular} & \begin{tabular}{|l|}
$-0,016461$ \\
\end{tabular} & 1 & 6,813 \\
\hline 37 & $\mathrm{X} 12$ & 0,3581 & 13,60576 & $-0,036640$ & 1 & $-0,0087807$ & 2,30113 & 0 & 0 & 6,563 \\
\hline 38 & $\mathrm{X} 13$ & 0,3059 & 8,49712 & $-0,032785$ & 1 & $-0,0002227$ & 2,97493 & 0 & 0 & 6,692 \\
\hline 39 & $\mathrm{X} 15$ & 0,2479 & 1,77343 & $-0,046817$ & 1 & $-8,8003 \mathrm{e}-9$ & 5,15361 & 0 & 0 & 6,810 \\
\hline
\end{tabular}

Пр имечания : $\mathrm{X} 08-x:=x+40 ; \mathrm{X} 30-x:=x+10 ; \mathrm{X} 04-x:=x+120 ; \mathrm{X} 29-x:=x+100$.
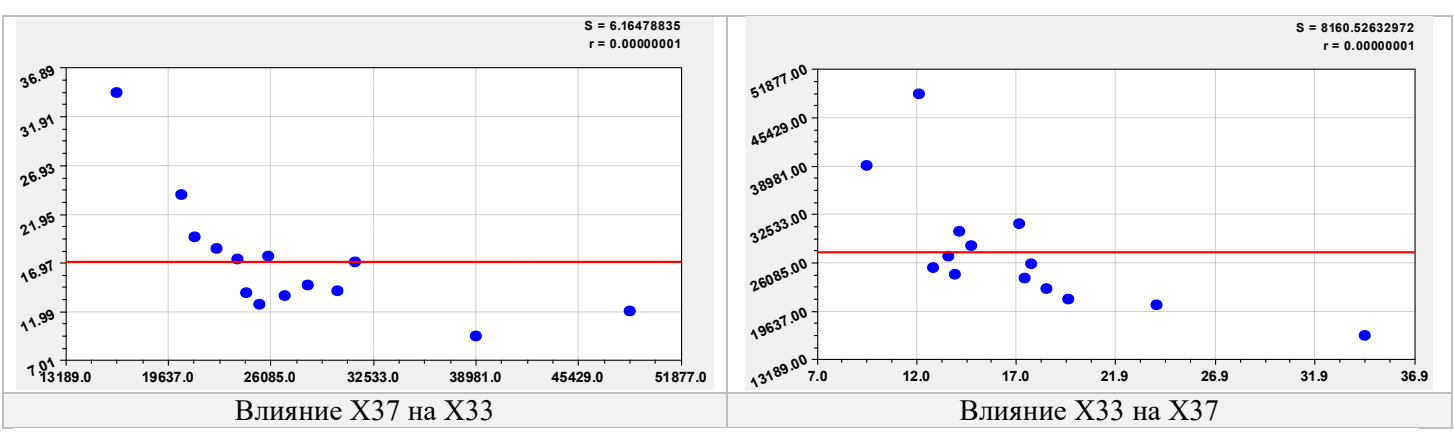

Рис. 3. Графики среднеарифметических значений двух показателей

При условии $c=1$ первая составляющая (1) превращается в закон Мандельброта (в физике), Лапласа (в математике), Перла Ципфа (в биологии) и Парето (в эконометрике). Переменная X27 получает модифицированный закон Мандельброта. При этом биотехнический закон в X27 идентичен форме кривой Кузнеца. При отрицательном знаке перед второй составляющей она становится кризисной, препятствующей росту показателя. При условиях $f=0$ и $g=0$ био- технический закон превращается в степенную функцию.

Оценка неравномерности иерархии из 39 влияющих параметров по стандартному отклонению показала, что разница между ними три раза.

Для случая многих показателей даже два из них имеют разные значения. Предлагаем коэффициент неравномерности, равный отношению стандартных отклонений формулы (1) и среднеарифметического $y_{0}=a$ (рис. 3 ). 
Для важнейшей пары Х37-Х33 (рис. 3) получаем среднееарифметическое $a=17,1 \%$, при этом по табл. $2 \mathrm{~S}=2,400 \%$. Из рис. 3 видно, что $\mathrm{S}_{0}=6,165$. Тогда коэффициент неравномерности $K$ для показателя $\mathrm{X} 33$ будет равен $K=2,400 / 6,165=0,3893$. А для пары Х33-Х37 (рис. 3) будет $a=27535,1$ руб/мес., а на рис. 2 видно, что $S=6284,3$ руб/мес., а на рис. 3 стандартное отклонение среднеарифметического равно $S_{0}=8160,5$ руб/мес. Коэффициент неравномерности будет равен $6284,3 / 8160,5=0,7701$. Тогда в $0,7701 /$ $0,3893=1,98 \approx 2$ раза по линии неравномерного распределения предпочтительнее пара X37-Х33 в сравнении с парой X33-X37.

Оказалось, что кривая Лоренца является в общем случае многокомпонентным уравнением, содержащим, кроме тренда, дополнительные колебания. Например, вейвлет-анализ динамического ряда температуры воздуха за 1959-2017 гг. дал 188 составляющих, из которых первые две составляющие относятся к тренду, а остальные 186 - к асимметричным колебаниям. При этом каждая составляющая является квантом поведения приземного слоя воздуха в процессе колебательной адаптации атмосферы. Причем все они распределяются фрактально по модифицированному закону Мандельброта по критерию стандартного отклонения.

\section{Распределение коэффициента коррелящии}

Далее рассмотрим, как распределяются влияющие 39 переменных по коэффициенту корреляции из табл. 1 внутри иерархии. При этом ранговое распределение X33 по двухсоставному тренду характеризуется коэффициентом корреляции 0,9940 . В табл. 1 дадим ему ранг 0 .

После идентификации вейвлета было получено (рис. 4) уравнение вида

$$
\begin{gathered}
r=r_{1}+r_{2}+r_{3}+\ldots, \\
r_{1}=0.99445 \exp \left(-9,935489 R^{0.62904}\right), \\
r_{2}=-9.37470 \cdot 10^{-8} R^{4.19717}, \\
r_{3}=A_{1} \cos \left(\pi R / p_{1}+0.80201\right), \\
A_{11}=4.88514 \cdot 10^{-59} R^{54.28608} \exp \left(-1,11738 R^{1.13496}\right), \\
p_{1}=-6.27817+0.19706 R^{1.32581} .
\end{gathered}
$$

Тренд является частным случаем вейвлета, поэтому из остатков видно, что возможно идентифицировать четвертое и последующие колебания. Первый член получает модифицированный закон Мандельброта, который показывает на фрактальное распределение переменных, влияющих на один показатель X33 - доля населения ниже прожиточного минимума, \%.

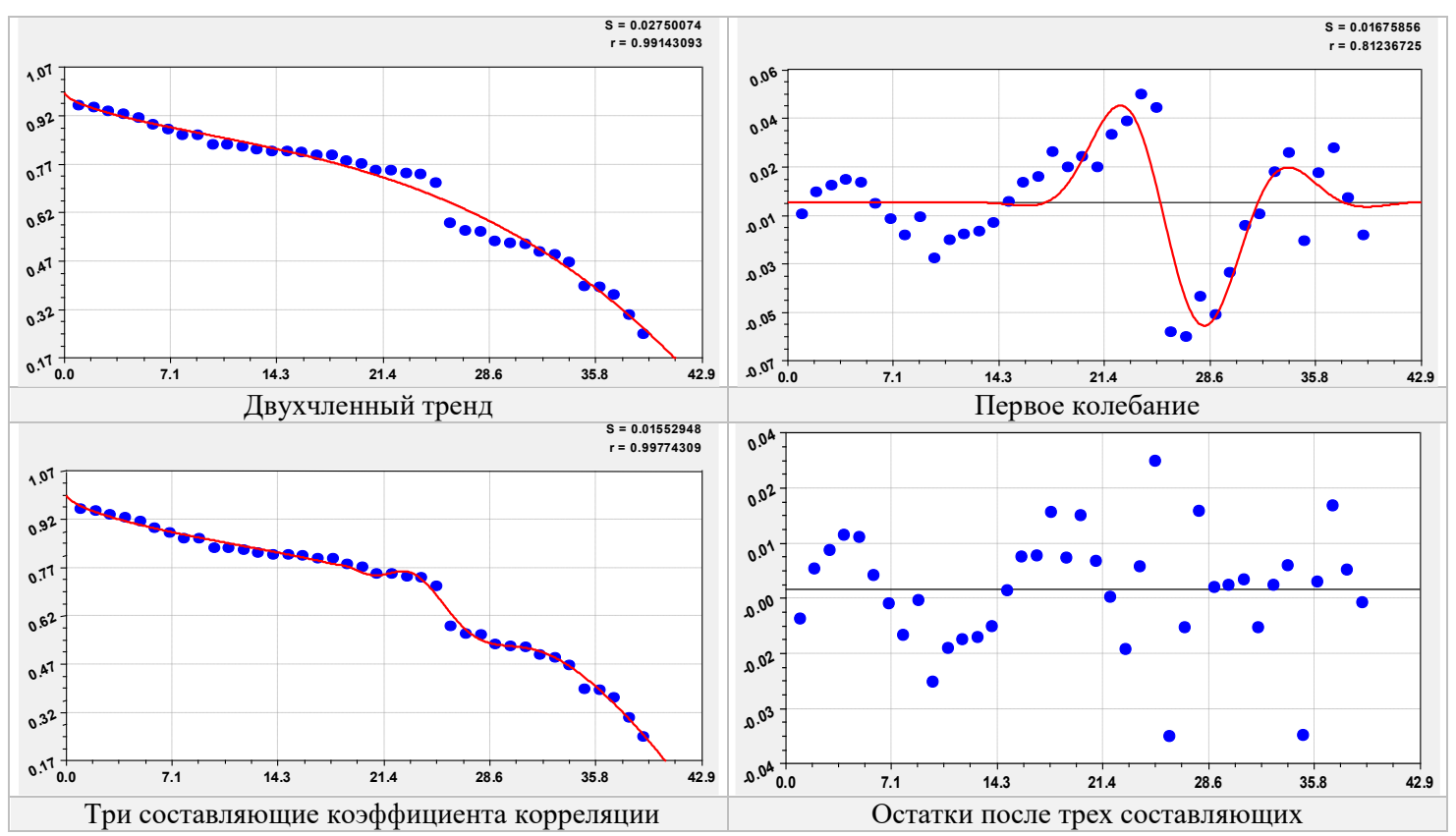

Рис. 4. Графики распределений коэффициента корреляџии по рангам в иерархии влияющих переменных 
В итоге доказана квантовая определенность параметров социумов в виде населения субъектов федерации. В дальнейшем возможна идентификация вейвлетов для всей России.

\section{Заключение}

Предложена система из 40 факторов жизнедеятельности населения 14 субъектов федерации Урала и Сибири, соотнесенная с географическими координатами (широтой и долготой) и высотой над уровнем Балтийского моря центров столиц этих же субъектов федерации. Обособленно рассмотрено влияние этих факторов как влияющих переменных на показатель в виде доли населения ниже прожиточного минимума. В факторном анализе этот показатель занял первое место в иерархии. Затем влияющие переменные были расставлены в рейтинге по убыванию коэффициента корреляции двухчленного тренда.

Выполнено сравнение кривой Лоренца по взаимообратному влиянию доходов населения (социумов) на долю ниже прожиточного минимума. Тогда коэффициент Джини неравномерности распределения превращается в статистические показатели в виде стандартного отклонения и коэффициентов корреляции.

Показано, что для коэффициента неравномерности пары переменные - показатель применяются стандартные отклонения, а для многих показателей учитывают отношения стандартных отклонений идентифицированной формулы к средней арифметической. Все закономерности имеют квантовую определенность в процессах колебательной адаптации социумов. Кванты поведения имеют фрактальные распределения по модифицированному нами закону Мандельброта.

Исследование выполнено при финансовой поддержке Российского фонда фундаментальных исследований, Правительства Красноярского края, Красноярского краевого фонда науки в рамках научного проекта: «19-45-240004p_a Прогноз эколого-экономического потенииала возможных «климатическиху миграциий в Ангаро-Енисейском макрорегионе в меняющемся климате 21го века».

\section{Список литературы / References}

1. Мазуркин П.М. Факторный анализ субъектов Сибирского округа по 40 параметрам жизни населения // Биосферное хозяйство: теория и практика. 2021. № 5 (35). С. 17-39.

Mazurkin P.M. Factor analysis of the subjects of the Siberian district by 40 parameters of the population's life // Biosphere economy: theory and practice. 2021. No. 5 (35). P. 1739 (in Russian).

2. Steffen W., Rockström J., Richardson K, Lenton T.M., Folke C., Liverman D., Summerhayes C.P., Barnosky A.D., Cornell S.E., Crucifix M., Donges J.F., Fetzer I., Lade S.J., Scheffer M., Winkelmann R. \& Schellnhuber H.J. Trajectories of the Earth System in the Anthropocene // PNAS, 2018. Vol. 115. No. 33. P. 8252-8259. DOI: 10.1073/pnas.1810141115.

3. Dovers S. \& Butler C. Population and environment: a global challenge - Curious // Earth \& environment. 2015. [Electronic resource]. URL: https://www.science.org.au/curious/earth-environment/population-environment (date of access: 09.09.2021).

4. Порфирьев Б.Н. «Зеленый» фактор экономического роста в мире и в России. [Электронный ресурс]. URL: porfirev-b.n.-zelenyj-faktor-ekonomicheskogo-rosta-v-mire-i-vrossii.pdf (дата обращения: 09.09.2021).

Porfiriev B.N. «Green» factor of economic growth in the world and in Russia. [Electronic resource]. URL: porfirev-b.n.zelenyj-faktor-ekonomicheskogo-rosta-v-mire-i-v-rossii.pdf (date of access: 09.09.2021)

5. Динамика доходов населения // Бюллетень о текущих тенденциях российской экономики. Вып. 58. Февраль 2020. 16 c.

Population income dynamics // Bulletin on current trends in the Russian economy. Issue 58. February 2020.16 p. (in Russian). 